\title{
HOMOTOPY NILPOTENCY AND ASSOCIATIVITY
}

ARTHUR H. COPELAND, JR. AND ALBERT O. SHAR

\begin{abstract}
If $(Y, \mu)$ is an $H$-space then a measure of nonassociativity, called Homotopy Nuclear Nilpotency (HNN) is defined. We then calculate the HNN of thirteen $H$-spaces: the twelve classes of multiplications on $S^{3}$ and the one class on $S_{(2)}^{5}$, the 5-sphere localized away from 2 .
\end{abstract}

1. If $(Y, \mu)$ is a homotopy associative $H$-space, Berstein and Ganea [B-G] defined $Y$ to have homotopy nilpotency less than $n$ if, for every space $X$, the group $[X, Y]$ is a nilpotent group of class less than $n$. It is easily seen that this is equivalent to the statement that $\beta_{n}: Y^{n} \rightarrow Y$ is homotopic to a constant map, where $\beta_{n}=\beta_{2}\left(\beta_{n-1} \times 1\right), \beta_{2}(x, y)=x^{-1} y^{-1} x y$ and $x^{-1}$ denotes the unique (up to homotopy) inverse of $x$. This second definition was used by Arkowitz and Curjel [A-C] and Gilbert [G] to extend the definition and calculations of homotopy nilpotency to the nonassociative multiplications on the $H$-spaces $S^{3}$ and $S^{7}$ where the homotopy inverse is well defined. Finally Zabrodsky [Z] defines homotopy nilpotency for an arbitrary $H$-space essentially by letting $\beta_{2}$ be such that $\mu\left(\beta_{2} \times \mu T\right) \Delta \sim \mu$. (Here $T$ is the twist map and $\Delta$ the diagonal.) In each of these cases the homotopy nilpotency of $Y$ gives some measure of the lack of commutativity of the multiplication $\mu$. However, if the multiplication $\mu$ on $Y$ is not associative, $[X, Y]$ forms an algebraic loop (not necessarily associative group) and for algebraic loops Bruck [B] has defined the concept of nuclear nilpotency as a measure of the nonassociativity of the loop. This definition may be worded as follows.

Let $G$ be a loop and $N$ a normal subloop (kernel). Define [G,N] to be the normal subloop of $G$ generated by all $x$ in $G$ which satisfy one of the equations in (1.1).

\section{CondiTion (1.1).}

$$
(c x)(a b)=(c a) b, a((c x) b)=(a c) b,(a b)(c x)=a(b c) \text { for } a, b \text { in } G, c \text { in } N .
$$

Define $\Gamma_{1}=G$ and $\Gamma_{n}=\left[G, \Gamma_{n-1}\right]$. Then $G$ is nuclear nilpotent of class $r$ if $\Gamma_{r+1}=1, \Gamma_{r} \neq 1$.

With this in mind we define the concept of Homotopy Nuclear Nilpotency.

Definition 1.2. An $H$-space $(Y, \mu)$ is homotopy nuclear nilpotent of class less than $n(\mathrm{HNN}(Y)<n)$ if for all spaces $X$ the loop $[X, Y]$ is nuclear nilpotent of class less than $n$. Thus $\operatorname{HNN}(Y)<2$ is equivalent to the statement that $Y$ is homotopy associative.

In $\$ 2$ we will prove the following theorem.

Received by the editors October 18, 1979.

1980 Mathematics Subject Classification. Primary 55P45.

(c) 1980 American Mathematical Society $0002-9939 / 80 / 0000-0573 / \$ 02.00$ 
THEOREM 1.3. For every multiplication on $S^{3}, H N N\left(S^{3}\right)<3$.

Note that of the 12 nonhomotopic multiplications on $S^{3}, 8$ are homotopy associative and for these the theorem is trivial. However, for the remaining four multiplications, Theorem 1.3 insures that they are as close to associative as possible. In $\$ 3$ we will calculate the HNN of $X=S_{(2)^{\prime}}^{5}$ (the 5-sphere $S^{5}$ localized away from 2).

If we replace the equations (1.1) with the equation $x(a c)=c a$ we can get a loop-theoretic definition of nilpotency which appears to correspond to Zabrodsky's topological definition. However while it is easy to show that $\beta_{n} \sim{ }^{*}$ implies the algebraic definition, the converse is an open question. Similarly there is, at present, no equivalent topological definition of homotopy nuclear nilpotency.

2. Nuclear nilpotency of $S^{3}$. Let $\left(S^{3}, \mu\right)$ be the $H$-space $S^{3}$ with the standard quaternionic multiplication. For any space $W$ let $\left[W, S^{3}\right]$ be the group of based homotopy classes of maps together with a product induced by this multiplication. Both of these products will be indicated by juxtaposition. Any of the 12 nonhomotopic multiplications $\mu_{r}, r=0,1, \ldots, 11$, induce a loop structure $\left[W, S^{3}\right]_{r}$ with the multiplication $\circ$ defined by $a \circ b=a b[a, b]^{r}$ where $[a, b]=a^{-1} b^{-1} a b$. By $[\mathbf{N}, 9.3]$ each of these loops is a Moufang loop (i.e., $(a \circ b) \circ(c \circ a)=(a \circ(b \circ c)) \circ a)$ and hence is diassociative (the subloop generated by any two elements is a group). Also note that $a \circ a^{-1}=1=a^{-1} \circ a$.

Let $a, b \in\left[W, S^{3}\right]_{r}$ and define the commutator by the formula $[a, b]_{r}=$ $a^{-1} \circ b^{-1} \circ a \circ b$. Diassociativity allows us to omit parentheses from this expression.

LEMMA 2.1. $[a, b]_{r}$ is in the center of the subgroup generated by $a$ and $b$.

Proof. The elements $a, b$ induce an element $(a, b)$ of $\left[W, S^{3} \times S^{3}\right]$. Then the image $(a, b)^{*}\left(\left[S^{3} \times S^{3}, S^{3}\right]_{r}\right)$ is a subloop of $\left[W, S^{3}\right]_{r}$ containing $a, b$. Further $(a, b)^{*}\left(\left[\left[p_{1}, p_{2}\right]_{r}, p_{1}\right]_{r}\right)=\left[[a, b]_{r}, a\right]_{r}$. But by $[\mathbf{N}, 5.8]\left[\left[p_{1}, p_{2}\right]_{r}, p_{1}\right]_{r}=1$. This implies the lemma by [B, VII, 5.1].

The following two corollaries are immediate.

Corollary 2.2. $[a, b]_{r}=\left[a^{-1}, b^{-1}\right]_{r},\left[a^{-1}, b\right]_{r}=[a, b]_{r}^{-1}$.

Corollary 2.3. [A-C, 4]. $[a, b]_{r}=[a, b]^{2 r+1}$.

For $a, b, c$ in $\left[W, S^{3}\right]_{r}$ the associator $[a, b, c]_{r}$ is defined by $(a \circ b) \circ c=$ $(a \circ(b \circ c)) \circ[a, b, c]_{r}$. In a manner similar to Lemma 2.1 we can prove

LEMMA 2.4. (a) $\left[[a, b, c]_{r}, a\right]_{r}=1$; (b) $\left[[a, c]_{r},[b, c]_{r}\right]_{r}=1$.

Proposition 2.5. Let $a, b, c \in\left[W, S^{3}\right]_{r}$. Then $[a, b, c]_{r}^{3}=1$.

We will give two proofs of Proposition 2.5, a completely algebraic one and a shorter topological one. The advantage of the algebraic proof is that it does not depend on the homotopy groups of $S^{3}$ and as such can, and will, be used for other $H$-spaces. 
Proof. It $r \neq 1 \bmod 3$ then $\left[W, S^{3}\right]_{r}$ is a group by [J1, Theorem (3.2)] and the result trivially holds.

If $r \equiv 1 \bmod 3$ then $[\mathrm{A}-\mathrm{C}],[\mathrm{B}]$ show that $\left[[a, b]_{r}, c\right]_{r}=1$. This combined with Lemma 2.4(a) and [B, VII, 5.5] imply

$$
[a \circ b, c]_{r}=[a, c]_{r} \circ[b, c]_{r} \circ[a, b, c]_{r}^{3} \text {. }
$$

Using Corollary 2.3 and the definition of $\circ$ we get

$$
\left[a b[a, b]^{r}, c\right]^{2 r+1}=[a, c]^{2 r+1}[b, c]^{2 r+1}\left[[a, c]^{2 r+1},[b, c]^{2 r+1}\right]^{r} \circ[a, b, c]_{r}^{3}
$$

By Lemma 2.4(b) we get

$$
\left[a b[a, b]^{r}, c\right]^{2 r+1}=[a, c]^{2 r+1}[b, c]^{2 r+1} \circ[a, b, c]_{r}^{3}
$$

But in any group $[[x y, z]]=[x, z][[x, z], y][y, z]$, so that

$$
\left[a b[a, b]^{r}, c\right]=[a b, c]\left[[a b, c],[a, b]^{r}\right]\left[[a, b]^{r}, c\right]=[a b, c][[a, b], c]^{r} \text {, }
$$

since $\left[[a b, c],[a, b]^{r}\right]=1$ and $[\mathrm{B}, \mathrm{VII}, 5.1]$. But $[a b, c]=[a, c][b, c][[a, c], b]$, since $[[a, c], b]$ is in the center of $\left[W, S^{3}\right]$.

Thus

$\left[a b[a, b]^{r}, c\right]=[a, c][b, c][[a, c],][[a, b], c]^{r}=[a, c][b, c][[a, b], c]^{r-1}$, since $[[a, c], b]=[[a, b], c]^{-1}[\mathrm{~B}, \mathrm{VII}, 5.3]$. Further $[\mathrm{B}, \mathrm{VII}, 5.3][[a, b], c]^{3}=1$. But $r \equiv 1 \bmod 3$ so that

$$
\begin{aligned}
{\left[a b[a, b]^{r}, c\right]^{2 r+1} } & =([a, c][b, c])^{2 r+1} \\
& =[a, c]^{2 r+1}[b, c]^{2 r+1}[[a, c],[b, c]]^{-(2 r+1) r} \\
& =[a, c]^{2 r+1}[b, c]^{2 r+1} \text { by }[\text { B, VII, 5.1] }
\end{aligned}
$$

Thus (2.7) becomes

$$
[a, c]^{2 r+1}[b, c]^{2 r+1}=[a, c]^{2 r+1}[b, c]^{2 r+1} \circ[a, b, c]_{r}^{3} \text { or }[a, b, c]_{r}^{3}=1 \text {. }
$$

Proposition 2.5 may be proven topologically by noting that the $\operatorname{map}[a, b, c]_{r}$ factors as

$$
W \stackrel{\Delta}{\rightarrow} W^{3} \rightarrow \Lambda^{3} W \rightarrow \Lambda^{3} S^{3}=S^{9} \rightarrow S^{3}
$$

and that $\pi_{9}\left(S^{3}\right)=Z / 3$.

LEMMA 2.8. $\left[[a, b, c]_{r}, d, e\right]_{r}^{2}=1$.

Proof. First note that $\left[[a, b, c]_{r}, d, e\right]_{r}=1$ if any of the entries are 1 so that $\left[[a, b, c]_{r}, d, e\right]_{r}$ factors as

$$
W \stackrel{\Delta}{\rightarrow} W^{5} \rightarrow \Lambda^{5} W \rightarrow \Lambda^{5}\left(S^{3}\right)=S^{15} \rightarrow S^{3} .
$$

But $\pi_{15}\left(S^{3}\right)=(Z / 2)^{2}$.

TheOREM 2.9. $\left[W, S^{3}\right]_{r}$ is nuclear nilpotental of class less than or equal to 2.

Proof. By the general techniques of $[\mathrm{B}]$ we need only show $\left[[a, b, c]_{r}, d, e\right]_{r}=1$. 
However

$$
\left[[a, b, c]_{r}, d, e\right]_{r}^{3}=\left[[a, b, c]_{r}^{3}, d, e\right]_{r}=1
$$

by [B, VII, 5.22] and Proposition 2.5 .

This combined with Lemma 2.8 completes the proof of 1.3 .

3. Nuclear nilpotency of $S_{(2)^{5}}^{5}$ Let $X=S_{(2)}^{5}$, be the localization of $S^{5}$ away from the prime 2. Then $X$ is an $H$-space and since $\pi_{10}(X)=0\left(\pi_{10}\left(S^{5}\right)=Z / 2\right)$, we note that $X$ has a unique (up to homotopy) $H$-structure and the multiplication is homotopy commutative.

Proposition 3.1. $X$ is not homotopy associative.

Proof. If $X$ were homotopy associative then $X$ would have a projective 3-space, $X P(3)$, so that $H^{*}(X P(3) ; Z / 3)=Z / 3[x] / x^{4}$ with dimension of $x$ equaling 6 . But by Theorem 2 of $[\mathbf{T}]$, this is impossible.

Proposition 3.2. Let $a, b, c \in[W, X]$, then $[a, b, c]^{3}=1$. This is an immediate consequence of Bruck's Theorem [B,VII, 5.5].

THEOREM 3.3. [ $W, X]$ is nuclear nilpotent of class 2.

Proof. As before we need only show that $\left[\left[a_{1}, a_{2}, a_{3}\right], a_{4}, a_{5}\right]=1$. Furthermore it is sufficient to demonstrate this when $W=\left(S^{5}\right)^{5}$ and $a_{i}: W \rightarrow S^{5}$ is the projection in the $i$ th component. But this map factors through $\Lambda^{5}\left(S^{5}\right) \rightarrow X$ and is the mapping $\langle\langle i, i, i\rangle, i, i\rangle$ defined by James [J2]. By Proposition $3.2\langle i, i, i\rangle=3 \alpha$ for some $\alpha \in \pi_{15}(X)=Z / 9$ so that $\langle\langle i, i, i\rangle, i, i\rangle=\langle 3 \alpha, i, i\rangle=3\langle\alpha, i, i\rangle$. But by Mimura [M-T] $\pi_{25}(X)=Z / 3\left(\pi_{25}\left(S^{5}\right)=Z / 6 \times Z / 2 \times Z / 2\right)$ and hence $\langle\langle i, i, i\rangle, i, i\rangle=$ 0 . This shows $N N P \leqslant 2$. Proposition 3.1 guarantees equality.

Observe that this proof imitates the algebraic proof of Proposition 2.5. The topological proof would be useless here, since it would involve factoring through $\pi_{15}(X)=Z / 9\left(\pi_{15}\left(S^{3}\right)=Z / 72+Z / 2\right)$.

\section{REFERENCES}

[A-C] M. Arkowitz and C. R. Curjel, Some properties of the exotic multiplications on the three-sphere, Quart. J. Math. Oxford Ser. (2) 20 (1969), 171-176.

[B-G] I. Berstein and T. Ganea, Homotopical nilpotency, Illinois J. Math. 5 (1961), 99-130.

[B] R. H. Bruck, A survey of binary systems, Ergebnisse der Math. und ihrer Grenzgebiete, Neue Folge, Heft 20, Reihe Gruppentheorie, Springer-Verlag, Berlin-Göttingen-Heidelberg, 1958.

[G] W. J. Gilbert, Homotopical nilpotence of the seven sphere, Proc. Amer. Math. Soc. 32 (1972), 621-622.

[J1] I. M. James, Multiplication on spheres. II, Trans. Amer. Math. Soc. 84 (1957), 545-558.

[J2]__ On H-spaces and their homotopy groups, Quart. J. Math. Oxford Ser. (2) 11 (1960), 161-179.

[M-T] M. Mimura and H. Toda, The $(n+20)$ th homotopy groups of $n$-spheres, J. Math. Kyoto Univ. 3 (1963), 37-58.

[N] C. W. Norman, Homotopy loops, Topology 2 (1963), 23-43.

[T] H. Toda, Note on cohomologying of certain spaces, Proc. Amer. Math. Soc. 14 (1963), 89-95.

[Z] A. Zabrodsky, Hopf spaces, North-Holland Math. Studies, Vol. 22, Notas de Matemática, No. 59, North-Holland, Amsterdam-New York-Oxford, 1976.

Department of Mathematics, University of New Hampshire, Durham, New Hampshire 03824 